\title{
A ROBUST METHOD TO RETRIEVE OPTION IMPLIED RISK NEUTRAL DENSITIES FOR DEFAULTABLE ASSETS
}

\author{
GUILLAUME LEDUC AND GREG OROSI
}

\begin{abstract}
Risk neutral densities recovered from option prices can be used to infer market participants' expectations of future stock returns and are a vital tool for pricing illiquid exotic options. Although there is a broad literature on the subject, most studies do not address the likelihood of default. To fill this gap, in this paper we develop a novel method to retrieve the risk neutral probability density function from call options written on a defaultable asset. The primary advantage of the method is that default probabilities inferred by the model can be analytically expressed and, if available, can be incorporated as an input in a flexible, robust and easily implementable manner.
\end{abstract}

\section{INTRODUCTION}

It is well-known that option prices contain information about the future prices of the underlying asset. In their seminal work, Breeden and Litzenberger (1978) illustrated that the discounted risk neutral density (RND) equals the second derivative of a European call option price with respect to its strike price. Therefore, given a series of options and their corresponding strikes, one can estimate the risk neutral density.

A robust method to extract RNDs is of great interest for academicians and practitioners. The connection between risk-neutral distributions and the market views of future prices was studied in Anagnou et al. (2002), Bahra (1997), and de Vincent-Humphreys and Noss (2012). Birru and Figlewski (2012) showed that exceptional detail about investors' expectations were provided by the RND of the S\&P500 during the melt down of fall 2008. Du et al. (2012) used RNDs to study the natural gas market. Aït-Sahalia and Lo (2000), Jackwerth (2000), Bliss and Panigirtzoglou (2004), Jackwerth (2004), and others demonstrated how RNDs reveal valuable insights about risk preferences of the market. Dupire (1994) revealed that under minimal conditions and

Corresponding author: Greg Orosi, American University of Sharjah, P.O. Box 26666, Sharjah, UAE. 
given a volatility surface it is possible to retrieve the unique diffusion process (and thus its RND), which is compatible with the volatility surface. Ross (2015) proved that under mild conditions, its is possible to fully recover the real world probability distribution corresponding to the RND. RNDs are needed to price illiquid exotic derivatives in a way that prevents static arbitrage opportunities.

Although one can estimate the risk neutral density from option prices using the Breeden-Litzenberger identity, in practice, a complete set of options prices is never available. Therefore, extracting well-behaved RNDs requires interpolation and extrapolation. This has attracted the attention of many researchers, and a vast literature exists on retrieving RNDs from observed option prices. For example, over 45 different methods are surveyed in Jackwerth (2004). Excellent surveys can also be found in Bahra (1997), Jondeau and Rockinger (2000), Skiadopoulos (2001), Monteiro et al (2008), Figlewski (2009), and Ludwig (2015). A comparison of the performance of non-parametric methods can be found in Lai (2012).

Figlewski (2009) highlighted that interpolation is typically performed in the implied volatility space, which involves fitting a spline or a loworder polynomial to the available data. A popular technique to do this task is to fit implied volatility-based models. Such techniques have excellent empirical performance, but Fengler (2009) and Monteiro et al (2011) pointed out that it is difficult to guarantee the absence of static arbitrage due to the non-linearity of the constraints in the related optimization problem. The resulting RND can take negative values and lead to mispricings. Other popular implied volatility-based models include the SABR model of Hagan et al. (2002), which is omnipresent in interest rate derivative pricing, and the Stochastic Volatility Inspired model (SVI) of Gatheral (2004). It should be noted that some implied volatility-based models can be calibrated in such a way that no static arbitrage violations occur (see for example the implementation of SVI by Gatheral and Jacquier (2013)); however, even these are not applicable to extracting RNDs from options on defaultable assets in a straightforward manner.

Clearly, retrieving RNDs from observed option prices has proved to be a challenging task. The reason for this is that finding an appropriate RND requires, as previously mentioned, the interpolation and extrapolation of call option prices in a way that satisfies several constraints:

(1) It must well fit the observed prices of the option;

(2) It must be free of static arbitrage; 
(3) It must smoothly interpolate observed option prices in spite of their noisiness and sparsity, avoiding overfitting,

(4) It must be flexible enough to allow for extrapolation of option prices in a fashion consistent with the views of the user, avoiding, for instance, imposing unrealistic fat tails on the RNDs;

(5) It must be easy to calibrate and implement;

(6) It must be flexible and adaptable to specificities of various markets, such as equities, commodities, currencies, interest rates, etc.

The purpose of this paper is to extend Orosi (2015a) to the case of a defaultable asset. Although the likelihood of default is a critically important feature of numerous asset classes, most techniques developed to extract RNDs fail to adequately address it. Our study is based on the novel parametric implementation of the framework of Orosi (2011) who presented a multi-parameter extension of the models of Figlewski (2002) and Henderson, Hobson, and Kluge (2007). Recent empirical studies Orosi $(2011,2015 \mathrm{a})$ support the use of this approach for its high empirical performance.

Although RNDs with a positive probability of default have been recently considered by Taylor et al. (2014), our work has several differences. First of all, a positive recovery can be incorporated into the model. Moreover, our framework does not make an explicit assumption about the form of the distribution and hence can be easily generalized. For example, models with a large number of parameters or nonparametric extensions are straightforward.

\section{Static Arbitrage-Free Conditions for Call Options}

Recall that the price $C(K, T)$ of a call option with strike $K$, maturity $T$, and risk free rate $r$ is given by

$$
\begin{aligned}
C(K, T) & =e^{-r T} E\left[\max \left(S_{T}-K, 0\right)\right] \\
& =e^{-r T} \int_{-\infty}^{\infty} \max \left(S_{T}-K, 0\right) p\left(S_{T}, T\right) d S_{T},
\end{aligned}
$$

where $p(\cdot, \cdot)$ is the risk neutral density of the asset $S_{T}$ at time $T$. Differentiating with respect to $K$, we obtain

$$
\frac{\partial C(K, T)}{\partial K}=-e^{-r T} E\left[\theta\left(S_{T}-K\right)\right]=-B(K, T),
$$


where $\theta(\cdot)$ is the Heaviside function and $B(K, T)$ is a European-style binary option with strike price $K$ and maturity $T$. It follows that

$$
-e^{-r T} \leq \frac{\partial C(K, T)}{\partial K} \leq 0
$$

since the price of a binary option with strike price $K$ is positive but has less value than a risk free payment of 1 at time $T$. Hence, the price of a call option is decreasing as a function of $K$.

Recall that

$$
B(K, T)=e^{-r T} P\left(S_{T}>K\right) .
$$

Hence, differentiating again with respect to $K$ gives

$$
\frac{\partial^{2} C(K, T)}{\partial K^{2}}=e^{-r T} p(K, T)
$$

since

$$
\frac{d}{d K} P\left(S_{T}>K\right)=\frac{d}{d K}\left(1-P\left(S_{T} \leq K\right)\right)=-p(K, T) .
$$

It follows that

$$
\frac{\partial^{2} C(K, T)}{\partial K^{2}} \geq 0
$$

Therefore, the price of the call option is convex as a function of strike $K$.

Finally, the price of a call option with zero strike is given by

$$
C(0, T)=e^{-r T} \int_{0}^{\infty} S_{T} p\left(S_{T}, T\right) d S_{T}=S_{0} e^{-d T},
$$

where $d$ is the continuous dividend yield.

It is known that there are several conditions that ensure no static arbitrage, but under minimal regularity assumptions allowing the differentiations above, conditions (2.1), (2.2) and (2.3) guarantee that at maturity $T$, the call prices are free of static arbitrage. In this paper, we focus on these conditions.

\section{Arbitrage-Free Call Option Pricing model}

Our approach closely follows that of Orosi (2011, 2015a) and assumes that the transformed call option price $c=C(K, T) /(S \exp (-d t))$ for a given maturity $T$ with strike price $K$ can be retrieved from the following equation:

$$
x=1-c+f(c)=1-c+\frac{F(c)}{c^{\alpha}}(1-c)^{\beta},
$$


where $x=K \exp (-r T) /(S \exp (-d T)), \beta \geq 1$, and $\alpha$ is a non-zero positive parameter. This representation with $F(c)=G, G$ a constant, $\beta=1$, and $\alpha=1$ was originally considered by Henderson, Hobson, and Kluge (2007) who extended a related model of Figlewski (2002). Henderson et al. point out that rather than attempting to determine suitable functions $c=f(x)$, it is easier to look for the inverse function $x=f^{-1}(c)$, where $f^{-1}(c)$ is a decreasing, convex function that satisfies $f^{-1}(c) \geq 1-c$ (the intrinsic value of call options). A function satisfying these conditions is graphed in the left part of Figure 1.

Given observed option prices $\left\{C_{i}\right\}_{i=1}^{n}$ at strike values $\left\{X_{i}\right\}_{i=1}^{n}$, the model's parameters can be determined by minimizing the following objective:

$$
\sum_{i=1}^{n}\left(c\left(x_{i}\right)-c_{i}\right)^{2},
$$

where $c_{i}=C_{i} /(S \exp (-d T)), x_{i}=X_{i} e^{-r T} /(S \exp (-d T))$, and $c\left(x_{i}\right)$, is determined numerically from

$$
x_{i}=1-c\left(x_{i}\right)+\frac{F\left(c\left(x_{i}\right)\right)\left(1-c\left(x_{i}\right)\right)^{\beta}}{c\left(x_{i}\right)^{\alpha}} .
$$

In this study, we use the following representation for $F(c)$ :

$$
F(c)=G(A c+1)^{\gamma} .
$$

Orosi (2015a) showed that this representation has the following advantages:

- The conditions (2.1), (2.2), and (2.3) are satisfied when the parameters are restricted to one of the following intervals:

$$
G>0, \alpha>0, \gamma>1,0>A>-1
$$

or

$$
G>0, \alpha>0,0>\gamma, A>0 .
$$

It is worthwhile to point out that, in the literature, several interpolants do not satisfy (2.3).

- If $\beta=1$, the model assumes a non-zero probability of default. In this study, we assume that $\beta=1$.

- The method is capable of extracting risk neutral densities with complex characteristics, such as bimodal distributions.

- The probability of default can be analytically calculated.

- The model can be easily generalized to incorporate more parameters. For example, considering

$$
F(c)=G(A c+1)^{\gamma} g(c)
$$


yields arbitrage-free call option prices as long as $g(c)$ is a positive, decreasing, and convex function.

\section{Calculating the Probability of Default}

With $\beta=1,(3.1)$ becomes

$$
x=1-c+\frac{F(c)}{c^{\alpha}}(1-c) .
$$

Implicitly differentiating this equation with respect to $x$, and making use of

$$
\begin{aligned}
B(K, T) & =-\frac{\partial C(K, T)}{\partial K} \\
& =-\frac{\partial(C)}{\partial x} \frac{\partial(x)}{\partial(K)} \\
& =-\frac{\partial\left(c S e^{-d T}\right)}{\partial x} \frac{\partial\left(\frac{K e^{-r T}}{S e^{-d T}}\right)}{\partial(K)} \\
& =-\frac{\partial c}{\partial x} e^{-r T},
\end{aligned}
$$

we obtain

$$
1=-\left(\frac{F(c)}{c^{\alpha}}+1\right) \frac{\partial c}{\partial x}+(1-c) \frac{d\left(\frac{F(c)}{c^{\alpha}}+1\right)}{d c} \frac{\partial c}{\partial x} .
$$

Substituting $c=1$ and using

$$
-\frac{\partial c}{\partial x}=e^{r T} B(0, T)=P\left(S_{T}>0\right)
$$

gives

$$
1=(F(1)+1) P\left(S_{T}>0\right) .
$$

It follows that the probability of the asset not defaulting prior to $T$ can be expressed as

$$
P\left(S_{T}>0\right)=\frac{1}{F(1)+1}=1-P D
$$

where $P D$ is the probability of default. 


\section{An Application to a Defaultable Stock}

To illustrate the applicability of our method, we calibrate our model to options on the General Motors stock with $T=1.63$ on May 30, 2008 , when the market saw a high probability of default before the option's expiry. After calculating option premiums using market observed volatilities with the Black-Scholes formula, our model was calibrated to the resulting European-style option values. The best fit parameters were obtained as follows: $G=0.4742, \alpha=0.4577, A=0.0121$, and $\gamma=-100$. Using (4.2), it can be inferred that $P D=12.5 \%$. The resulting cumulative distribution and probability density are shown in Figures 2 and 3, respectively.

\section{Incorporating the Probability of Default}

Often, the probability of default $P D$ can be reliably estimated; see Finkelstein (2001) for instance. When this is the case, $P\left(S_{T}>0\right)=$ $1-P D$ is given, and equation (4.2) can be rearranged to yield the following restriction on parameter $G$ :

$$
G=\frac{P D}{(1-P D)(A+1)^{\gamma}} .
$$

Hence, if the probability of the asset defaulting by time $T$ is known $a$ priori, it can be incorporated into our model as a parameter. This approach is illustrated by calibrating the model to previously considered General Motors stock options with various default probability values. The resulting cumulative distributions and probability densities are shown in Figures 4 and 5, while the resulting call option values are depicted in Table 2. Although the different values of default probabilities lead to almost identical European call option prices, it can be observed from Figure 4 that the resulting binary prices vary significantly. Hence, when probabilities of defaults are available, exotic European options can be priced with higher accuracy.

\section{Non-Zero Recovery}

In this section, we consider the case when the value of the stock in the case of default is given by $R$, a non-zero value. Moreover, Chang and Orosi (2016) show that

$$
C(K, T)=\left\{S e^{-d T}-K e^{-r T} \text { if } K \leq R .\right.
$$

Then, the transformed call prices must satisfy 


$$
c=1-x
$$

when $K \leq R$, or equivalently,

$$
x=1-c,
$$

and transformed call, $c^{*}$, at $K=R$ is given by

$$
c^{*}=1-R \exp (-r T) /(S \exp (-d T)) .
$$

Then, letting $c^{*}=1-R \exp (-r T) /(S \exp (-d T))$, the function

$$
x=\left\{\begin{array}{ll}
1-c & \text { if } K \leq R \\
1-c+G \frac{\left(c^{*}-c\right)}{c^{\alpha}} & \text { if } K>R
\end{array},\right.
$$

is convex, monotone, and continuously differentiable as long as $G>0$, and $\alpha>0$. Moreover, it can be shown that $P D$ is given by

$$
\begin{aligned}
P D= & 1-P\left(S_{T}>R\right)= \\
& 1-\frac{1}{1+\frac{G}{\left(c^{*}\right)^{\alpha}}} .
\end{aligned}
$$

Therefore, using our model with (7.2) in lieu of (4.1), one can retrieve an RND in the presence of positive recovery. Orosi (2015b) points out that the options on General Motors stock on April 15, 2009 must have assumed a non-zero recovery because the stock price was $S=1.89$ and the value of the option at $K=1$ is given by (7.1) (see Table 3). To illustrate the applicability of our method, we calibrated our model with $R=1$. The best fit parameters were obtained as follows: $G=0.4671$ and $\alpha=0.8979$ that imply that $P D=47.8 \%$.

\section{Conclusion}

In this paper, we employ a new, flexible, robust, and easy to implement method to calculate the risk neutral densities extracted from call options written on a defaultable asset. An interesting feature of our method is that default probabilities can be expressed analytically or, when available, incorporated as an input. To demonstrate the applicability of the method, we fit the model to vanilla options written on General Motors stock. Our results show that the probability default has a significant impact on the resulting binary options and little impact on the vanilla option prices. We additionally showed how our model can be extended to incorporate a positive recovery that can also be inferred by the model or given as an input. 


\section{REFERENCES}

[1] Aït-Sahalia, Y. and Lo, A. W. "Nonparametric Risk Management and Implied Risk Aversion." Journal of Econometrics, 94 (2000) pp 9-51.

[2] Anagnou, I., Bedendo, M., Hodges, S., Thompkins, R. "The relation between implied and realised probability density functions." Working paper, University of Technology, Vienna, (2002).

[3] Bahra, B. "Implied Risk-Neutral Probability Density Functions From Option Prices: Theory and Application." Bank of England Working Paper Series (1997).

[4] Benaim, S., Dodgson, M., and Kainth, D. "An arbitrage-free method for smile extrapolation." Working Paper, QuaRC, Royal Bank of Scotland, (2008).

[5] Black, F., and Scholes, M.S. "The Pricing of Options and Corporate Liabilities." Journal of Political Economy, 81 (1973) pp. 637-59.

[6] Bliss, R. and Panigirtzoglou, N. "Option-implied risk aversion estimates." The Journal of Finance, 59 (2004), pp. 407-446.

[7] Breeden, D., and Litzenberger, R. "Price of State-Contingent Claims Implicit in Options Prices." Journal of Business, 51 (1978), pp. 621-651.

[8] de Vincent-Humphreys, R., Noss, J. "Estimating probability distributions of future asset prices: empirical transformations from option-implied risk-neutral to real-world density functions." Bank of England Working Paper, (2012).

[9] Chang, B.Y., and Orosi, G. "Equity Option Implied Probability of Default and Equity Recovery Rate" Working Paper, Available at SSRN: http://ssrn.com/abstract=2698831, (2016).

[10] Du, Y. Wanga, C., and Dub, Y. "Inversion of option prices for implied riskneutral probability density functions: general theory and its applications to the natural gas market." Quantitative Finance 12 (2012), pp 1877-1891.

[11] Dupire B. "Pricing with a Smile." RISK 7 (1994) pp. 18-20.

[12] Fengler, M.R. "Arbitrage-Free Smoothing of the Implied Volatility Surface." Quantitative Finance, 9 (2009), pp. 417-428.

[13] Figlewski, S. "Assessing the Incremental Value of Option Pricing Theory Relative to an Informationally Passive Benchmark." The Journal of Derivatives, Fall 2002, pp. 80-96.

[14] Birru, J. and Figlewski, S. "Estimating the implied risk neutral density for the U.S. market portfolio." Volatility And Time Series Econometrics: Essays In Honor Of Robert F. Engle, Tim Bollerslev, Jeffrey R. Russell and Mark Watson, eds., Oxford, UK: Oxford University Press (2008).

[15] Figlewski, S. "Anatomy of a meltdown: The risk neutral density for the S\&P500 in the fall of 2008."Journal of Financial Markets 15 (2012), pp. 151180.

[16] Finkelstein, V. "Assessing Default Probabilities from Equity Markets." Presentation, Credit Risk Summit, New York (2001).

[17] Gatheral, J. "A parsimonious arbitrage-free implied volatility parameterization with application to the valuation of volatility derivatives." Presentation at Global Derivatives \& Risk Management, Madrid (2004).

[18] Gatheral, J. and Jacquier, A. "Arbitrage-free SVI volatility surfaces." Available at SSRN 2033323 (2013).

[19] Hagan, P. S., Kumar, D., Lesniewski, A. S. and Woodward, D. E. "Managing Smile Risk", Wilmott Mag. September (2002) pp. 84-108. 
[20] Henderson, V., Hobson, D., and Kluge, T. "Is There an Informationally Passive Benchmark for Option Pricing Incorporating Maturity?." Quantitative Finance, 7 (2007), pp. 75-86.

[21] Heston, S.L. "A Closed-Form Solution for Options with Stochastic Volatility with Applications to Bond and Currency Options." The Review of Financial Studies 6 (1993) pp. 327-343.

[22] Jackwerth, J.C. "Recovering risk aversion from option prices and realized returns." Review of Financial Studies. 13 (2000) pp. 433-451.

[23] Jackwerth, J.C. "Option-Implied Risk-Neutral Distributions and Risk Aversion." Research Foundation of AIMR, Charlotteville (2004).

[24] Jondeau, E. and Rockinger, M. "Reading the smile: the message conveyed by methods which infer risk neutral densities." Journal of International Money and Finance 19 (2000), pp. 885-915.

[25] Lai, W. "Comparison of Methods to Estimate Option Implied Risk Neutral Densities" Quantitative Finance, Forthcoming.

[26] Ludwig, M. "Robust Estimation of Shape Constrained State Price Density Surfaces." Journal of Derivatives, Spring 2015, 22, pp. 56-72.

[27] Monteiro, A.M., Tütüncü, R.H. Reha., and Vicente, L.N. "Recovering Risk neutral Probability Density Functions from Options Prices using Cubic Splines and Ensuring Nonnegativity." European Journal of Operational Research, 187, 525-542 (2008).

[28] Monteiro, A.M., Tütüncü, R.H., and Vicente, L.N. "Estimation of risk neutral density surfaces", Computational Management Science, 8, 4 , pp. 387-414 (2011) .

[29] Orosi, G. "Improved Implementation of Local Volatility and Its Application to S\&P 500 Index Options." The Journal of Derivatives, Spring 2010, pp. 53-64.

[30] Orosi, G. "A Multi-Parameter Extension of Figlewski's Option-Pricing Formula.", Journal of Derivatives, Fall 2011, pp. 72-82.

[31] Orosi G. "Estimating Option-Implied Risk-Neutral Densities: A Novel Parametric Approach", Journal of Derivatives, Fall 2015, pp. 41-61.

[32] Orosi, G. "Closed-Form Interpolation-Based Formulas for European Call Options Written on Defaultable Assets", Journal of Asset Management,16 (2015) 236-242.

[33] Ross, S. "The Recovery Theorem", The Journal of Finance, 70, 2, pp. 615-648 (2015).

[34] Skiadopoulos, G. "Volatility smile consistent option models: a survey." International Journal of Theoretical and Applied Finance 4, pp. 403-437 (2001).

[35] Taylor, S.J., Tzeng, C., and Widdicks M. "Bankruptcy Probabilities Inferred from Option Prices.", Journal of Derivatives, Winter 2014, pp. 8-31. 